\title{
Exertional Rhabdomyolysis: A Case of Markedly Elevated Creatine Kinase Without Acute Kidney Injury
}

\author{
Andrew C. Berry ${ }^{\mathrm{a}, \mathrm{g}}$, Joshua D. Lenchus ${ }^{\mathrm{b}}$, Danny Avalos ${ }^{\mathrm{c}}$, Ariel Caplan ${ }^{\mathrm{d}}$, \\ Bruce B. Berry ${ }^{\mathrm{e}}$, Michael J. Monzel ${ }^{\mathrm{f}}$
}

\begin{abstract}
Rhabdomyolysis describes a clinical syndrome in which striated muscle breaks down and intracellular contents are released into the circulation. Exertional rhabdomyolysis can be caused by a new, intense workout routine or a change in intensity of an existing one. The severity of presentation varies from asymptomatic creatine kinase (CK) elevations to life-threatening renal failure, disseminated intravascular coagulation, cardiac dysrhythmias and/or compartment syndrome. Our case describes a patient with exertional non-traumatic rhabdomyolysis caused by a mild workout routine change with markedly elevated CK level. There was no evidence of any concomitant renal failure or other aberrancies, such as compartment syndrome, electrolyte abnormalities or electrocardiogram changes. Although the patient reports this as his initial episode of such symptoms, inherited metabolic disorders and genetic mutations should be considered and further workup performed. To the best of our knowledge, this is a rare case of a significantly elevated CK resulting from modest exercise that did not result in acute kidney injury.
\end{abstract}

Keywords: Rhabdomyolysis; Acute kidney injury; Renal failure; Creatine kinase; Physical activity

Manuscript accepted for publication May 6, 2014

${ }^{a}$ Kansas City University of Medicine and Biosciences, Kansas City, MO, USA

${ }^{\mathrm{b}}$ Department of Internal Medicine, University of Miami Miller School of Medicine, Jackson Memorial Hospital, Miami, FL, USA

${ }^{c}$ Department of Internal Medicine, University of Miami Miller School of Medicine, Regional Campus, Atlantis, FL, USA

${ }^{\mathrm{d}}$ Department of Internal Medicine, Palm Beach Centre for Graduate Medical Education, West Palm Beach, FL, USA

${ }^{\mathrm{e}}$ Department of Internal Medicine, Wheaton Franciscan Healthcare, Milwaukee, WI, USA

${ }^{f}$ Department of Gastroenterology, Digestive Disease Center of the Palm Beaches, Loxahatchee, FL, USA

${ }^{g}$ Corresponding Author: Andrew C. Berry, Kansas City University of Medicine and Biosciences, 1750 Independence Ave, Kansas City, MO 64106, USA. Email: ABerry5555@gmail.com

doi: http://dx.doi.org/10.14740/jmc1792w

\section{Introduction}

Rhabdomyolysis describes a clinical syndrome in which striated muscle breaks down and releases intracellular contents into the circulation, often presenting with at least five to tenfold elevations in serum creatine kinase (CK) [1]. The severity of presentation varies from asymptomatic CK elevations to life-threatening renal failure, compartment syndrome, disseminated intravascular coagulation and/or cardiac dysrhythmias [2-4]. The etiology of rhabdomyolysis can be broken down into following groups: non-exertional nontraumatic (e.g., toxins, drugs, electrolyte imbalances, acute infections), exertional non-traumatic (e.g., marked exertion in untrained individuals or under strenuous conditions, metabolic myopathies, hyperthermia) and traumatic (crush injury, extensive immobilization). The cause can also be multi-factorial in nature. Patients commonly present with the triad of myalgias, dark urine and weakness, though the presence of all three is not required for diagnosis. Clinical manifestations include myoglobinuria, myalgias, elevated serum CK levels, abnormal liver enzymes and electrolyte abnormalities. The goal of intervention is to prevent or treat acute kidney injury. Therefore, vigorous intravenous (IV) hydration is the mainstay of therapy. In addition, correction of electrolyte abnormalities and alkalinization of urine with sodium bicarbonate are utilized.

\section{Case Report}

A 28-year-old Caucasian male without significant past medical history presented to the emergency department with "dark urine and muscle pains". The patient reported starting a new upper body workout 2 days prior that involved circuit training consisting of $1 \mathrm{~h}$. Despite being an avid runner, he had not previously participated in weight training exercises. After his first workout, the patient experienced upper extremity myalgias that he considered "normal wear and tear". He awoke the next day with moderate to severe myalgias, yet proceeded to exercise again, repeating a similar upper extremity workout as he had done the previous day. His body 
continued to ache after the second workout and he awoke on the day of admission with what he described as "non-bloody, dark urine". The patient drank water, but continued to experience the dark urine. Remaining concerned, he sought medical attention at the emergency department.

The patient did not report any significant past surgical history, medication use or allergies. He did not relate any consumption of alcohol, tobacco or illicit drug use. Family history was unremarkable for genetic disorders, coagulopathies or myopathies. Aside from that reported in the history of present illness, his review of systems was otherwise negative. Electrocardiogram (ECG) demonstrated normal sinus rhythm with narrow-complex tachycardia. Vital signs show blood pressure $167 / 100$, temperature $36.8^{\circ} \mathrm{C}$, pulse 123 , respiration rate 18 , and $100 \%$ oxygen saturation on room air. Physical exam was positive for tenderness to palpation of the upper extremities, bilaterally, and diffusely in all abdominal quadrants. Laboratory data on admission showed $\mathrm{CK}$ $92,803 \mathrm{U} / \mathrm{L}$, white blood cell $14,400 \mathrm{~mm}^{3}$, blood urea nitrogen $(\mathrm{BUN}) 14$, and creatinine $(\mathrm{Cr})$ 1.01. Dipstick urinalysis revealed $3+$ blood, urine myoglobin of 750 , specific gravity $<1.005$, and trace protein and ketones. The patient was admitted with the diagnosis of rhabdomyolysis, and received multiple doses of $1,000 \mathrm{~mL} 0.5 \%$ sodium chloride and sodium bicarbonate at $200 \mathrm{~mL} / \mathrm{h}$.

Serum myoglobin level on admission was elevated at 18,781. CK peaked at $97,472 \mathrm{U} / \mathrm{L}$ on the night of admission. On hospital day 3, liver function tests were significantly elevated: AST 1,711, ALT 457, total alkaline phosphate 80, but CK decreased to 70,912 U/L, aldolase 0, BUN 5, Cr 0.7, with resolved leukocytosis and normal electrolytes. Hepatitis panel was negative. The patient remained in the hospital for a total of 6 days and remained on IV fluids containing sodium bicarbonate. Upon discharge, AST and ALT were trending down, $\mathrm{CK}$ was $14,580 \mathrm{U} / \mathrm{L}$, and the patient was able to tolerate oral fluids.

\section{Discussion}

Exertional rhabdomyolysis can be caused by a new, intense workout routine or a change in intensity of an existing one, and represents approximately one-third of reported cases of rhabdomyolysis [5]. Recently, there has been a rising concern of exertional rhabdomyolysis within Crossfit ${ }^{\mathrm{TM}}$ classes, which involves spontaneous high intensity exercises unfamiliar to athletes using a traditional exercise regimen [6].

This patient's presentation of exertional rhabdomyolysis is rather unique. As an avid runner, averaging 15 miles per week, he was hardly a novice athlete. He had no known prior medical conditions, major electrolyte abnormalities, recent infections, or recent consumption of supplements, alcohol or toxins. The patient routinely engaged in repetitive exercises (e.g., push-ups, sit-ups), was a young male, and exercised under peer pressure of an athletic trainer, all variables increasing the likelihood of exertional rhabdomyolysis [7-12]. Though exercise and strength training does produce a transient rise in CK levels [1], the intensity of the patient's new exercise regime in a climate-controlled environment and his negative past medical history does not adequately explain such an extreme rise in his CK $(97,472 \mathrm{U} / \mathrm{L})$. In a review of patients with exertional rhabdomyolysis as the sole etiology, the average CK was 40,471 IU/L [5]. The literature suggests the rate of acute kidney injury in patients with exertional rhabdomyolysis is much less (19.1\%) than rhabdomyolysis from other causes (34.2\%) [5]. Though there is a modest predictive correlation between $\mathrm{CK}$ levels and the development of acute renal failure, with levels higher than 16,000 $\mathrm{U} / \mathrm{L}$ more likely to be associated with, one might expect this patient to have significantly higher serum creatinine levels or significant oliguria [13]. This patient is unique due to his markedly elevated CK level, lack of concomitant renal failure or other aberrancies, such as compartment syndrome, electrolyte abnormalities or ECG changes. Although the patient reports this as his initial episode of such symptoms, inherited metabolic disorders and genetic mutations should be considered.

Metabolic myopathies, genetic mutations and inherited disorders of metabolism can present with rhabdomyolysis. The most common metabolic myopathies include myophosphorylase deficiency (McArdle disease), carnitine palmitoyl transferase deficiency, and adenosine monophosphate deaminase deficiency [1]. Notably, this patient never reported a prior incident of myalgias or urine discoloration following a workout. Though some of the aforementioned metabolic diseases can have late onset in the second or third decade of life, one might have expected the patient to suffer from repeated episodes. Similarly, several mutations and polymorphic variations in other human genes, heat shock proteins, and interleukin- 6 have been associated with exertional muscle damage, heat intolerance and exaggerated post-exercise $\mathrm{CK}$ levels. One study reported that $23 \%$ of patients with recurrent exertional rhabdomyolysis had enzyme defects and another $18 \%$ with other muscle diseases, muscular dystrophies or myopathies [14]. However, over $50 \%$ of patients with recurrent exertional rhabdomyolysis had no known biochemical cause [14]. A clinician should consider further testing on a case-by-case basis.

There is much debate on what diagnostic studies are appropriate given the nature of exertional rhabdomyolysis and its suggested low recurrence rate [15]. Most practitioners recommend further testing only if the patient has recurrent episodes, persistently elevated serum CKs, or develops evidence of acute kidney injury unresponsive to IV hydration. However, due to the relatively mild to moderate exertional nature of onset and marked CK level, further metabolic and genetic workup in this particular patient may be warranted. To the best of our knowledge, this is a rare case of a signifi- 
cantly elevated CK resulting from modest exercise that did not result in acute kidney injury.

\section{References}

1. Landau ME, Kenney K, Deuster P, Campbell W. Exertional rhabdomyolysis: a clinical review with a focus on genetic influences. J Clin Neuromuscul Dis. 2012;13(3):122-136.

2. Tsai CN, Liu MF, Lin TS, Lin LH, Wang CR. Rhabdomyolysis and acute renal failure in a polymyositis patient. Mod Rheumatol. 2004;14(5):422-423.

3. Melli G, Chaudhry V, Cornblath DR. Rhabdomyolysis: an evaluation of 475 hospitalized patients. Medicine (Baltimore). 2005;84(6):377-385.

4. Warren JD, Blumbergs PC, Thompson PD. Rhabdomyolysis: a review. Muscle Nerve. 2002;25(3):332-347.

5. Alpers JP, Jones LK, Jr. Natural history of exertional rhabdomyolysis: a population-based analysis. Muscle Nerve. 2010;42(4):487-491.

6. Hak PT, Hodzovic E, Hickey B. The nature and prevalence of injury during CrossFit training. J Strength Cond Res. 2013.

7. Frucht M. Challenge, 110 deep knee bends; reward, rhabdomyolysis. N Engl J Med. 1994;330(22):16201621 .

8. Mahakkanukrauh A, Sangchan A, Mootsikapun P. Exertional rhabdomyolysis following excessive exercise of university freshman cheer-training. J Med Assoc Thai. 2003;86(8):789-792.

9. Juray RM. Exertional rhabdomyolysis in unsupervised exercises in a correctional setting: a case study. Urol Nurs. 2005;25(2):117-119.

10. Kruse DH. "Tyson squats" as a cause of rhabdomyolysis. J Emerg Nurs. 1998;24(2):116-117.

11. Granata A, Lo Piccolo G, Ruffo C, Vittoria S, Stalteri A. Rhabdomyolysis after body building exercise. Nephron. 2002;91(2):354-355.

12. Springer BL, Clarkson PM. Two cases of exertional rhabdomyolysis precipitated by personal trainers. Med Sci Sports Exerc. 2003;35(9):1499-1502.

13. Ward MM. Factors predictive of acute renal failure in rhabdomyolysis. Arch Intern Med. 1988;148(7):15531557.

14. Lofberg M, Jankala H, Paetau A, Harkonen M, Somer H. Metabolic causes of recurrent rhabdomyolysis. Acta Neurol Scand. 1998;98(4):268-275.

15. Krivickas LS. Recurrent rhabdomyolysis in a collegiate athlete: a case report. Med Sci Sports Exerc. 2006;38(3):407-410. 